\title{
A Research on Spatial Distribution and Spatiotemporal Evolution of Population in Jiangsu Province
}

\author{
Chang Jiang \\ College of Geographic and Biological Information \\ Nanjing University of Posts and Communications \\ Nanjing, P.R. China \\ jiangc@njupt.edu.cn
}

\author{
Yunsong Shi \\ English Department \\ Nanjing University of Chinese Medicine \\ Nanjing, P.R. China \\ shiyunsong@njutcm.edu.cn
}

\begin{abstract}
Based on demographic data in Jiangsu Province from 2004 to 2009 , the characteristic and law of spatial population distribution in Jiangsu Province was analyzed with population density analysis, spatial auto-correlation, spatial construction index analysis and gravity center analysis. The results show that: (1) the population density is obviously distinct in spatial, which appears dense in the center of city and gradually reduces in the country around downtown area; the most areas appears dense lie in the Southern Jiangsu and Central Jiangsu; ( 2 ) population distribution in Jiangsu Province significantly shows spatial clustering pattern on the whole, where in the part shows well spatial construction and form the southeast - northwest patterns; ( 3 ) the gravity center of population moved to the northwest, which migrated along with economic center; ( 4 ) population distribution in Jiangsu Province appears imbalance; though the end of populations distribution shows concentration, the amplitude of changes slows down.
\end{abstract}

Index Terms-population density, spatial autocorrelation, spatial construction index, gravity center.

\section{INTRODUCTION}

As an important population and socioeconomic phenomenon, population distribution refers to the distribution of population in a certain region in a certain number and size. It is the manifestation of spatial distraction of population during the course of population development and also the core issue of population geography[1]. From 1970s to 1980s, with the application of quantitative methods to the empirical researches, many spatial analyses were viewed as the key in numerous population geography researches. Rees and Wilson argue that the task of population geography lies in the integration of spatial analysis and human demography[2]. In recent years, most researchers applied this integrated method in the research on population distribution. Nie Qin applied GIS to the research on the population distribution and its spatial correlation in more than 108 counties in Shandong Province [3].By analyzing the data of population landscape density in Shenyang based on grid, Du et al. applied the theories and methods in geographic statistics into the analysis to the spatial relevance and variability so as to explore out the applicability of the population density model in classic cities in a micro level[4]; With the help of the GIS software platform, Wang et al. applied the raster cost weighted distance method to the analysis of the relationship between the population distribution and the accessibility in the larger area transportation in China[5]; $\mathrm{Wu}$ et al. introduced the regional gravity center model to measure and analyze the balance of Jiangsu regional development[6];Han et al. used LORENZE curve method to analyze the uneven distribution of population in China[7]; By using multiple quantity methods like centralization index in population distribution, Gini coefficient and population index, Zhang Jingjing made a comprehensive study in the urban and suburban population distribution in the North Slope Area in Tian Mountain[8]. The above studies all analyzed the local population distribution differences from a certain spatial dimension, but none analyzed the spatial distribution and its spatiotemporal evolution from different dimensions. Based on this founding, this paper applies the population density method and spatial auto-correlation method to analyze Jiangsu's population distribution from the dimension of counties and also analyzes the spatiotemporal evolution from the dimension of prefecture-level cities with the help of gravity center analysis and spatial pattern index. With this research, the authors expect to provide a certain kind of reference to the decisions of the sustainable development in Jiangsu's economy, population and environment.

\section{STUDY AREA AND DATA}

\section{A. Research Area}

Jiangsu Province lies in the middle of coastal areas to the west of Yellow Sea, and the north of Yangtze River Delta. Jiangsu borders Zhejiang Province and Shanghai in the southeast, Anhui Province in the west and Shandong in the North. It is located between latitudes $30^{\circ} 45^{\prime} \mathrm{N}$ and $35^{\circ} 20^{\prime} \mathrm{N}$ and between longitudes $116^{\circ} 18^{\prime} \mathrm{E}$ and $121^{\circ} 57^{\prime} \mathrm{E}$.With a land borderline of $3,383 \mathrm{~km}$, Jiangsu covers an area of $10.26 \mathrm{~km}^{2}$, $10 \%$ of the national area but the area per capita is the least among all the provincial divisions in China. Jiangsu is divided into thirteen prefecture-level divisions. The capital city is Nanjing. The population of Jiangsu is 78,988,000 ranking as the fifth in China. Due to the natural, social and economical 
differences, Jiangsu has distinctive economic development patterns in Southern Jiangsu, Central Jiangsu and Northern Jiangsu. Besides, this paper uses the data from the old administrative units to analyze to avoid possible wrong results.

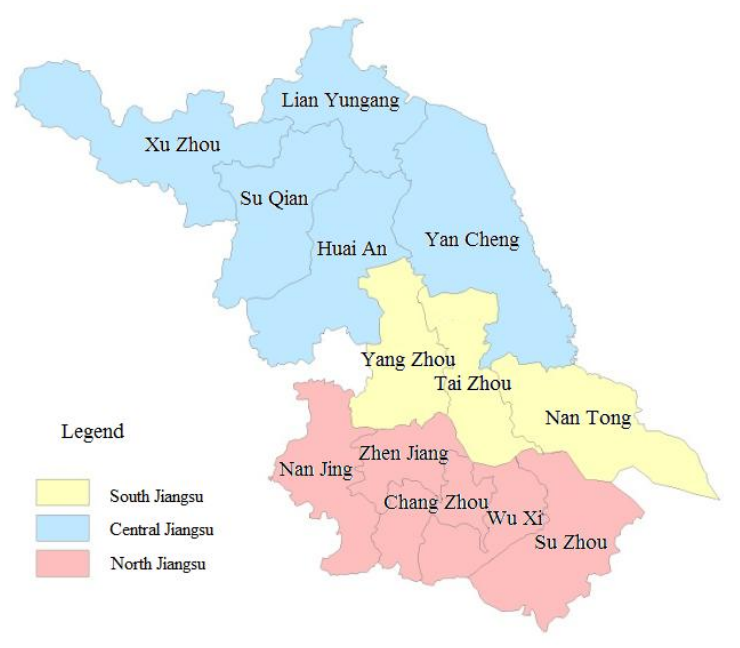

Fig. 1. Economic sketch map of Jiangsu in 2008

\section{B. Data Processing}

According to Jiangsu Statistics Almanac in 2008, Jiangsu has 13 prefecture-level cities, 54 urban districts, 27 county-level cities and 25 counties. Suzhou, Wuxi, Changzhou, Nanjing and Zhenjiang along with the districts and counties subordinate to them consist of Southern Jiangsu; Yangzhou, Taizhou and Nantong with the districts and counties subordinate to them consist of Central Jiangsu; Xuzhou, Huaian, Suqian, Yancheng and Lianyungang with their boroughs and counties--Northern Jiangsu[9].

From the prefecture-level city and also county or countylevel cities dimension, this paper analyzed the differences in regional population distribution in Jiangsu. The data collected in this paper range from 2004 to 2009 in 65 districts or counties in each prefecture-level city from official publication. The population figures are based on census registered; the economic figures are based on Jiangsu GDP statistics. Because some data are missing due to the change of the administrative division, this paper only chooses the complete and representative data in the spatial distribution analysis.

\section{METHODOLOGY}

\section{A. Population Density Analysis}

The population density is a guideline to demonstrate and measure the population distribution difference[10]. The population density $d$ can be defined as in

$$
d=\frac{P}{S}
$$

where $\mathrm{P}$ refers to population, and $\mathrm{S}$ refers to the land area. What we need to note is that this calculating methods works only provided that the population is even distributed in a region and the density cannot reflect the real spatial distraction of population because the density is calculated on the basis of a random choice of the region (an administrative division or population census district). Hence this paper uses the interpolation method to get access to the population density contour map in Jiangsu.

\section{B. Analysis on the Spatial Autocorrelation}

Jiangsu population is uneven distributed. The spatial patter tends to be stable, less possible to change drastically in a short run. So Global Spatial Autocorrelation Indices and local spatial autocorrelation analysis are necessary to be introduced in this research. With Global Moran's I and Local Moran's I, this paper explores out the spatial patterns of the regional population distribution as well as the spatial relations and differences inside and outside each district.

\section{1) Global Moran's I}

Global Moran's I was proposed by Moran in 1948, which refers the similarity of the properties of two spatially neighboring areas.

$$
I=\frac{n}{\sum_{i=1}^{n} \sum_{j=1}^{n} w_{i j}} \times \frac{\sum_{i=1}^{n} \sum_{j=1}^{n} w_{i j}\left(x_{\mathrm{i}}-\bar{x}\right)\left(x_{\mathrm{j}}-\bar{x}\right)}{\sum_{i=1}^{n}\left(x_{\mathrm{i}}-\bar{x}\right)^{2}}
$$

In $\bar{x}=\frac{1}{n} \sum_{i=1}^{n} x_{i}, \mathrm{n}$ means the number of the administrative districts, $x_{1}$ and $x_{2}$ are the measured value of property $X$ in spatial unit $i$ and $j \cdot \bar{x}$ is the average value and $w_{i j}$ is the spatial weight matrix made on the basis of the neighboring standard.

\section{2) Local Moran's I}

Local Moran's I refined the patter and process of the space with help of the four quadrants in Moran's scatter chart and is widely applied in the statistic analysis in area features. This index discloses four spatial relations to four different spatial patters respectively. The upper right quadrant (high-high autocorrelation): the high clustering area is surrounded by high clustering neighbor; The upper left quadrant (low-high autocorrelation): the low clustering area is surrounded by high clustering neighbor; The bottom right quadrant (high-low autocorrelation): the high- centralized area is surrounded by low clustering neighbor; The bottom left quadrant (low-low autocorrelation): the low clustering area is surrounded by low clustering neighbor. High-high autocorrelation and low-low autocorrelation areas share small differences from the neighboring areas; low-high and high-low area share more differences. 


$$
I_{i}=\frac{\left(x_{\mathrm{i}}-\bar{x}\right)}{\sum_{i=1}^{n}\left(x_{\mathrm{i}}-\bar{x}\right)^{2}} \times \sum_{j=1}^{n} w_{i j}\left(x_{\mathrm{j}}-\bar{x}\right)
$$

In the above, the meaning of variants is identical to what in Eqs. (2). What Local Moran's $I$ has measured are all correlation of $X$ in spatial unit $i$ and $j$.

\section{3) Gravity Center Analysis}

This paper introduces gravity center in the research, namely, with the balance point that the population distribution and the economy distribution reached in spatial torque in the research area in a certain time, we can analyze this area's evolution in the population distribution and economy distribution; with the moving track and speed of population gravity center or economy center, we can disclose the characteristics and causes of the changes of the research area's population and economy distribution[14][15]. $\bar{x}$ and $\bar{y}$ are written as

$$
\bar{x}=\frac{\sum_{i=1}^{n} p_{i} x_{i}}{\sum_{i=1}^{n} p_{i}}, \bar{y}=\frac{\sum_{i=1}^{n} p_{i} y_{i}}{\sum_{i=1}^{n} p_{i}},
$$

where $n$ means the number of the administrative unit, and $\left(x_{i}, y_{i}\right)$ are the geographic gravity center of each basic unit. This paper defines $p_{i}$ as the population number and local GDP of this basic unit and $(\bar{x}, \bar{y})$ is the population gravity and economic center in Jiangsu.

\section{4) Balance Analysis}

In order to investigate whether Jiangsu population spatial pattern is relatively clustered or balanced, this paper uses uneven index $(U)$ and centralized index $(C)$ in this research[16][17]. The uneven index is written as

$$
U=\sqrt{\frac{\sum_{i=1}^{n}\left[\frac{\sqrt{2}}{2}\left(x_{i}-y_{i}\right)\right]^{2}}{n}},
$$

where $n$ means the number of the administrative districts, $x_{i}$ means the proportion of City $i$ population in the whole province's population. $y_{i}$ is the proportion of city I's population in the whole population; the smaller $U$ is, the more balanced the population spatial pattern is . The centralized index is written as

$$
C=\frac{1}{2} \sum_{i=1}^{n}\left|x_{i}-y_{i}\right|,
$$

where $n, x_{i}, y_{i}$ share the same meanings as shown in Eqs. (5); $\mathrm{C}$ ranges from 0 to 1 and the smaller $\mathrm{C}$ is, the deflection of population's cluster to one particular area is less and the population distribution pattern is more balanced while the bigger $\mathrm{C}$ is, the distribution is more centralized.

\section{RESULTS AND ANALYSIS}

\section{A. Jiangsu population density shows a regional difference of gradual decrease from the north to the south.}

This paper takes the demographic data of 13 prefecturelevel cities in Jiangsu in 2008 to make the Jiangsu Population Density Distribution Map. Compared to the Jiangsu population density map in , Fig. 1 shows the population density in Jiangsu from 2004 to 2009 increased gradually, with 724 persons $/ \mathrm{km}^{2}$ in 2004 while 753 persons $/ \mathrm{km}^{2}$ in 2009. Central Jiangsu covers the least area with the biggest population density while Northern Jiangsu the largest area and smallest density, even lower than the provincial average value. The areas with big population density mainly lie in Southern and Central Jiangsu, whose economies is better and the urban population and whose density is bigger than the neighboring cities or even prefecture-level cities.

TABLE I. JiANGSU POPULATION DENSITY CHART FROM 2004 To 2009( persons / $\mathrm{km}^{2}$ )

\begin{tabular}{|c|c|c|c|c|}
\hline \multirow{2}{*}{ Year } & \multicolumn{4}{|c|}{ Region } \\
\cline { 2 - 5 } & Jiangsu & $\begin{array}{c}\text { Southern } \\
\text { Jiangsu }\end{array}$ & Central Jiangsu & $\begin{array}{c}\text { Northern } \\
\text { Jiangsu }\end{array}$ \\
\hline 2004 & 724 & 800 & 847 & 617 \\
\hline 2005 & 729 & 810 & 846 & 598 \\
\hline 2006 & 736 & 821 & 847 & 603 \\
\hline 2007 & 743 & 829 & 845 & 607 \\
\hline 2008 & 748 & 832 & 844 & 608 \\
\hline 2009 & 753 & 837 & 844 & 612 \\
\hline
\end{tabular}

By comparing the evolution of the population density in 13 prefecture-level cities in Jiangsu from 2004 to 2009, this paper finds that the density figures only shrink in Yancheng and Nantong, the two coastal cities while the other 11 cities all increase more or less. According the degree of the increase in the population density, Nanjing ranks as the first followed by Suzhou, Wuxi, Xuzhou, Lianyungang, Changzhou, Suqian, huaian, Zhenjiang,Yangzhou and Taizhou. The top three all lie in Southern Jiangsu due to the better transportation and economy. Northern Jiangsu has a small density due to its weak economy and less influence from the economically advanced regions. 


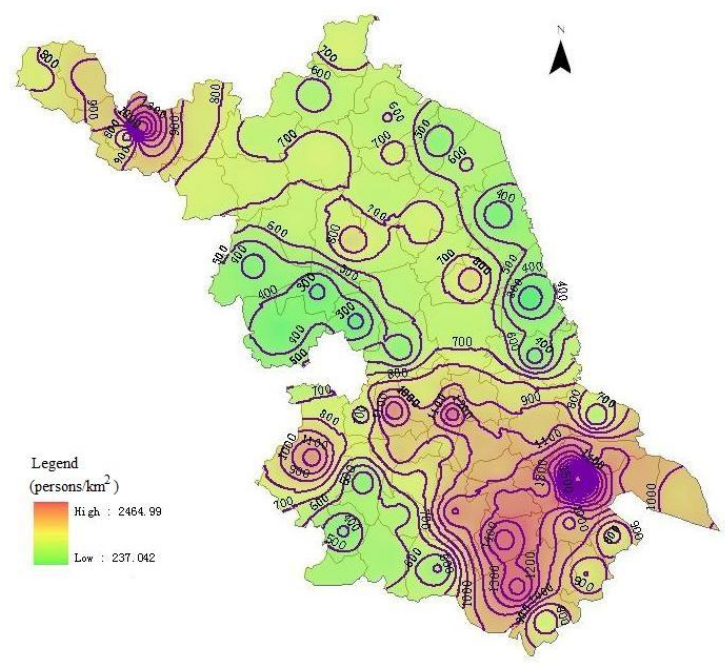

Fig.2. Jiangsu population desity map in 2008

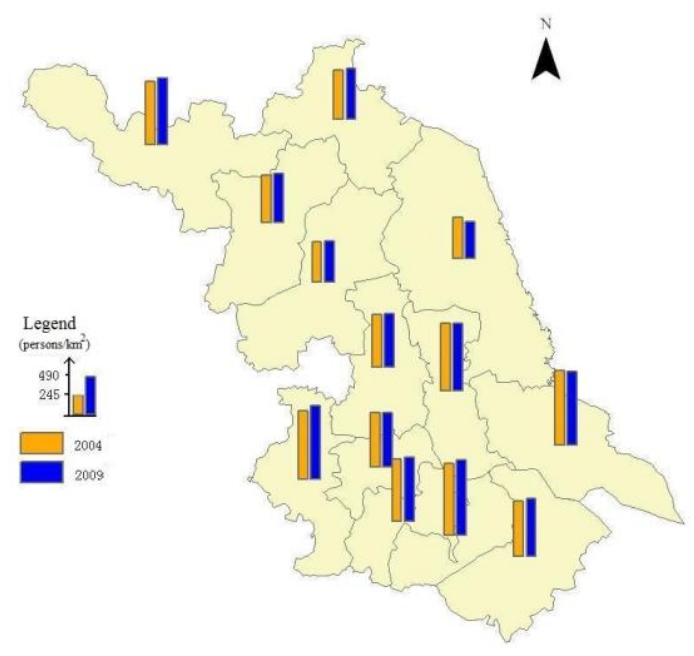

Fig.3. The evolustion of Jiangsu population desity

B. The population distribution in Jiangsu shows a significant spatial pattern of high centralization in Southern and Central Jiangsu and low centrlization in Northern Jiangsu.

With the analysis that Global Moran's I made to Jiangsu population density, we first presumes no spatial autocorrelation exists among the 65 research units in Jiangsu from 2004 and 2009. Then we gets a significance level $a=0.05$ and on this level the author tests the presumption and gets a critical value $\mathrm{Z}_{0.05}=1.96$. All Global Moran's I values in Jiangsu population density from 2004 to 2009 are bigger than 1.96 and passes the significant tests. Table II shows Jiangsu population spatial patter is a centralized one in general, namely, whether the area with big population density or the one with small density shows a certain aggregation in geography and small density area neighbors small density areas while big density area neighbors big density ones. The six years' evolution of Global Moran's I seems stable which shows that the spatial distribution of population density doesn't change a lot.

TABLE II. THE MORAN'S INDEX STATISTICS OF JIANGSU POPULATION DENSITY FROM 2004 TO 2009

\begin{tabular}{|c|c|c|c|}
\hline \multirow{2}{*}{ Year } & \multicolumn{3}{|c|}{ Statistics Value } \\
\cline { 2 - 4 } & Moran's $\boldsymbol{I}$ & $\boldsymbol{S t d}$ & $\boldsymbol{Z}$ \\
\hline 2004 & 0.2585 & 0.0523 & 5.24 \\
\hline 2005 & 0.2554 & 0.0516 & 5.25 \\
\hline 2006 & 0.2521 & 0.0513 & 5.22 \\
\hline 2007 & 0.2509 & 0.0511 & 5.22 \\
\hline 2008 & 0.2529 & 0.0518 & 5.18 \\
\hline 2009 & 0.2502 & 0.0517 & 5.14 \\
\hline
\end{tabular}

By analyzing Local Moran's I, Jiangsu population density shows a pattern of high-high and low-low autocorrelation, which indicates the spatial cluster pattern and its distribution features of Jiangsu population density. The bigger population density in Jiangsu mostly lies in Central and Southern Jiangsu (significantly highclustering) while the lower density lies in Northern Jiangsu. Central and Southern Jiangsu has a high-high autocorrelation due to their natural and economic development. This area is one of the wealthiest areas in Yangtze Delta and even the whole China, famous for its "Southern Jiangsu Development Pattern". The speedy and healthy development of Southern Jiangsu's economy needs sufficient labor forces, attracts massive labor force and therefore develops into a high-clustering pattern. Northern Jiangsu lies in the junction of Jiangsu, Shandong, Henan and Anhui Province with a weaker economic condition. For a long time, it is less affected and radiated by advanced economic central areas. Therefore, this area absorbs less labor force and the population density is smaller. 


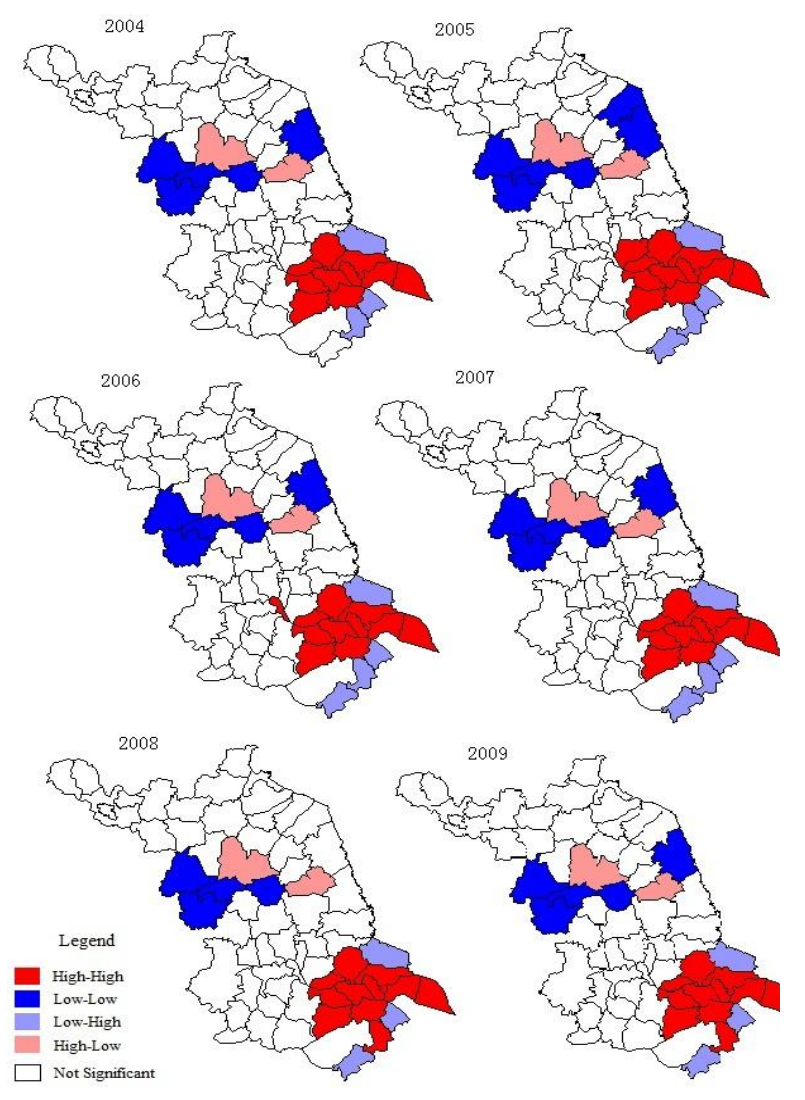

Fig.4. The local spatial autocorrelation pattern of Jiangsu population density from 2004 to 2009

C. The gravity center of population in Jiangsu moves northweat along with the economic gravity center

Table III the population gravity center coordinate and economic center of each year in Jiangsu from 2004 to 2009 . Fig.7 shows that Jiangsu population gravity center is always located in Gaoyou, a county-level city in central part of Yangzhou, deviated away from the central south of Yangzhou, the geographic center of Jiangsu Province. In 2004, the distance between the population gravity center and the geographic center was $14.58 \mathrm{~km}$, but in 2009 , it is 14.87 . This is mainly due to the influence from Northern Jiangsu, especially Xuzhou, which is constantly leading in population number. Yancheng is next to Xuzhou. This two driving force makes the population gravity center moves to the northwest in general.
TABLE III. THE DEVIATION DISTANCE BETWEEN JIANGSU POPULATION GRAVITY CENTER(PGC), ECONOMY GRAVITY CENTER(EGC) AND GEOGRAPHIC GRAVITY CENTER(GGC)

\begin{tabular}{|c|c|c|c|c|c|}
\hline \multirow{2}{*}{ Year } & \multicolumn{5}{|c|}{ Deviation Distance } \\
\cline { 2 - 6 } & $\begin{array}{c}\text { Between } \\
\text { PGC and } \\
\text { GGC } \\
(\boldsymbol{k m})\end{array}$ & $\begin{array}{c}\text { Eorth } \\
\text { Eetween } \\
\text { GGC } \\
(\boldsymbol{k m})\end{array}$ & $\begin{array}{c}\text { and } \\
\text { South } \\
\text { Distance } \\
\text { between } \\
\text { PGC and } \\
\text { EGC } \\
(\boldsymbol{k m})\end{array}$ & $\begin{array}{c}\text { East and } \\
\text { West } \\
\text { Distance } \\
\text { between } \\
\text { PGC and } \\
\text { EGC } \\
(\boldsymbol{k m})\end{array}$ & $\begin{array}{c}\text { Between } \\
\text { PGC and } \\
\text { EGC } \\
(\boldsymbol{k m})\end{array}$ \\
\hline 2004 & 14.58 & 60.31 & 69.30 & 27.50 & 74.56 \\
\hline 2005 & 14.49 & 62.65 & 71.45 & 28.40 & 76.89 \\
\hline 2006 & 14.57 & 63.23 & 71.85 & 29.20 & 77.56 \\
\hline 2007 & 14.50 & 63.43 & 71.87 & 29.60 & 77.73 \\
\hline 2008 & 14.65 & 62.4 & 70.93 & 29.60 & 76.86 \\
\hline 2009 & 14.87 & 59.89 & 68.68 & 29.10 & 74.59 \\
\hline
\end{tabular}

Figure 5 and Table III show a gradual northwestward movement tendency. The distance in a straight line of the population center deviation in the course of the six years is $1.2 \mathrm{~km}$, for the stability of the population spatial pattern determines that the population movement is extremely slow. The factors that affect this area's population mobility are reproduction and migration of population. Northern Jiangsu has a large population increase rate and population base and the increase tends to develop to northwest. However, due to the developed economy, the population of Southern and Central Jiangsu tend to move southward. Hence, the pulling force of different directions leads to balance and the population in the end tends to move westward in general.

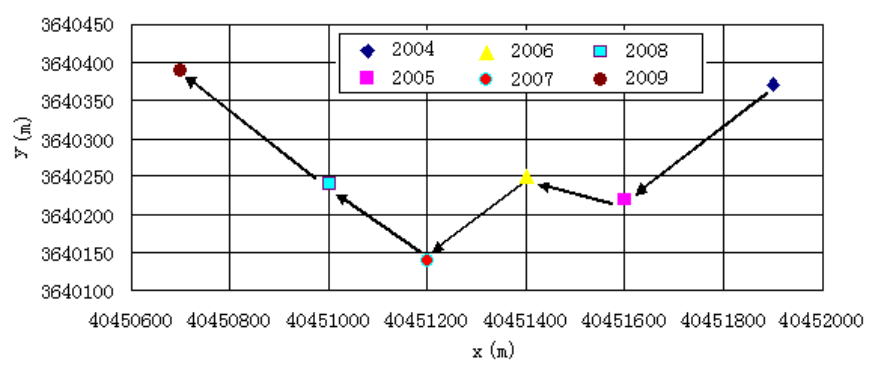

Fig.5. The moving track of Jiangsu population gravity center

Jiangsu's economic gravity center mostly lies in Yangzhou, a county-level city in the south of Yangzhou. The economic gravity center is away in the southeast from the geographic center. This shows that the economic spatial pattern in the southeast of Jiangsu is obviously better than that in the northwest of Jiangsu. Most economy-advanced areas are clustered in the basic industrial zone along the Yangtze River but different districts enjoy different levels. Separated by the year of 2007, the moving track of economic gravity center have two stages: Stage one, deviation to the southeast with a distance of $3.1 \mathrm{~km}$ from 2004 to 2007; Stage two, deviation to the northwest with a distance of $3.6 \mathrm{~km}$. The moving track of 
the economic gravity center shows that regional economy in Jiangsu is being evenly developed. During the "11th Five-year Plan" time, Northern Jiangsu achieved an economic development and its comprehensive economic power significantly increased which lead the northwestward deviation of the economic gravity center.

The common place of the movement track between Jiangsu population gravity center and economic gravity center lies in the northward deviation, which is mainly related to the development of the "Major-Three Area". From 2004 to 2007 including the "11th Five-Year Plan" period, the increase of GDP in Southern Jiangsu is higher than that in Northern Jiangsu while from 2007 to 2009, the latter is higher than the former. This indicates that Jiangsu plan in a more overall range. "south-to-north Cooperation", a typical example worked effectively and increased Northern Jiangsu's economic power so as to lead to the northwestward migration of the population gravity center along with the economic gravity center.

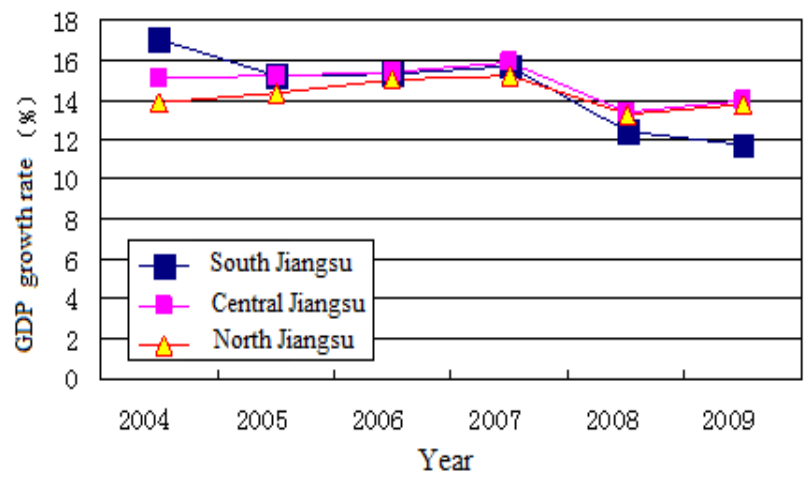

Fig.6. GDP increase rate from 2004 to 2009 in Jiangsu

The deviation of Jiangsu's population gravity center is bigger in the distance away from the south-to-north direction than from the east-to-west direction. Take 2009 as an example, the population and economic gravity center is deviated $68.68 \mathrm{~km}$ away from the south-to-north direction while $29.1 \mathrm{~km}$ away from the east-to-west direction. This indicates that the differences in population distribution and economic development pattern are larger from the south-to-north direction than that from the east-to-west direction. Compared with the data in 2004, the population and economic gravity center is deviated less than $0.62 \mathrm{~km}$ in the south-to-north direction, while more than $1.6 \mathrm{~km}$ from the east-to-west direction. This shows Jiangsu's development were achieved by both the south and north regions in a new phase. Admittedly, the gradient gap in regional development, the overall development level in Northern Jiangsu seems to be smaller and smaller than that in Southern Jiangsu.

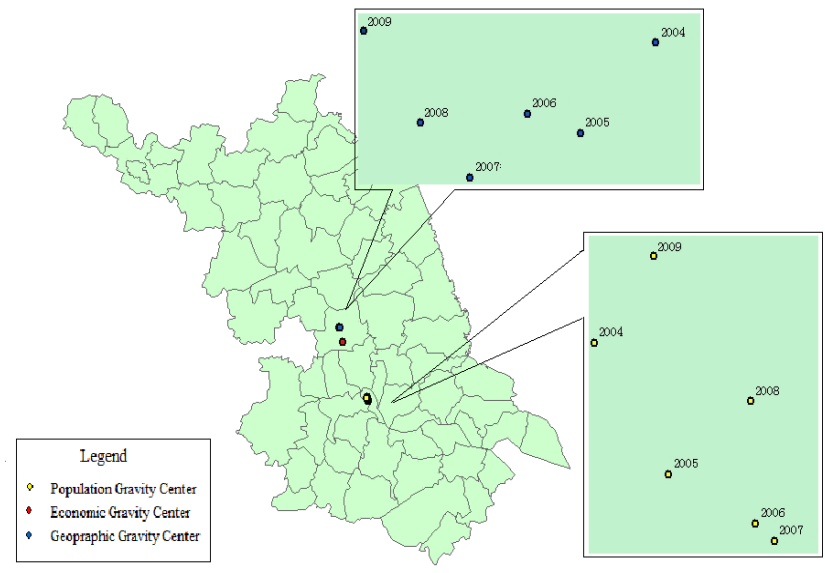

Fig.7. Jiangsu population, economic gravity center and geopraphic gravity center

D. The centralization trend in Jiangsu's spatial distraction of population tend to be slow.

After analyzing the uneven index and centralization index, Table IV shows that Jiangsu spatial distraction of population witnessed a process of centralization-decentralizationcentralization. The overall distribution tends to be centralized but in a slow way. From 2004 to 2006, the population centralization index increases rapidly by a rate of 0.003 per year; from 2007 to 2009, the centralization index increases by a rate of 0.0002 , which indicates a slow speed of population migration to Southern Jiangsu.

TABLE IV. JIANGSU POPULATION SPATIAL PATTERN INDEX FROM 2004 TO 2009

\begin{tabular}{|c|c|c|}
\hline Year & Uneven Index (u) & Centralization Index (c) \\
\hline 2004 & 0.012531 & 0.091443 \\
\hline 2005 & 0.014873 & 0.100741 \\
\hline 2006 & 0.014825 & 0.100807 \\
\hline 2007 & 0.014825 & 0.101402 \\
\hline 2008 & 0.014828 & 0.101393 \\
\hline 2009 & 0.014932 & 0.102104 \\
\hline
\end{tabular}

\section{DiscuSSSION AND CONCLUSION}

This paper applies the methods of population density and spatial auto-correlation to analyze Jiangsu's population distribution patter from the dimension in a county level. By using the gravity analysis method and spatial pattern index, the authors get an access to the features of the spatial evolution of population distribution from the dimension in a prefecture-level city angle. Based on Jiangsu's population distribution pattern and its spatiotemporal evolution from different spatial dimensions from 2004 to 2009, this paper concludes as follows: 
- Jiangsu's population density has a significant regional difference decreasing from the Southern Jiangsu to the Northern Jiangsu. The central city urban areas are the most populous while the surrounding counties or county-level cities are gradually less populous. Most populous districts are located in Southern and Central Jiangsu.

- Jiangsu's population distribution has a significant centralization pattern in an overall view and a good spatial pattern in a local spatial analysis view.

- Jiangsu's rapid economic development and unbalanced regional development lead to the bigger economic gravity center deviation. Compared with economic gravity center, population gravity center is less deviated. The distance of the deviation in population and economic gravity center increases from the the east-north direction while decreases from south-tonorth direction. This shows Jiangsu's development were achieved by both the south and north regions in a new phase. Admittedly, the gradient gap in regional development, the overall development level in Northern Jiangsu seems to be smaller and smaller than that in Southern Jiangsu.

- Jiangsu's spatial distraction of population witnesses a process of centralization-decentralizationcentralization due to the governmental regulation during the "11th Five-Year-Plan" period. Jiangsu population is uneven distributed in geography. Though it tends to be centralized, the trend is becoming slower and slower.

\section{ACKNOWLEDGMENT}

This research was supported in part by the Nanjing University of Posts and Communications for Laboratory Construction and Equipment Management Research Project, Grant 2012XSG16, for Qinglan Project, Grant NY208039, and for Key Bidding Project in Teaching Reform, Grant JG03212JX02.

\section{REFERENCES}

[1] Hu.H.Y and Zhang.S.Y. Population geography of China.Shanghai:East China Normal University Press, 1984.

[2] Zhu Junmin, "The progress of population geography," World Regional Studies. vol. 1, pp.80-86, 1994.
[3] Nie qin, "A study on population and spatial autocorrelation in Shandong province," Science of Surveying and Mapping.vol.36,pp.216,217,223, Mar 2011.

[4] Du Guoming,Zhang Shuwen,and Zhang Youquan, "Analyzing spatial auto-correlation of population distribution: a case of Shenyang city," Geographical Research,vol.26,pp. 383-390,Mar 2007.

[5] Wang Zhenbo,Xu Jiangang,Zhu Chuangeng, Qi Yi,and Xu Lu, "The country accessibility divisions in China and its correlation with population distribution," Geographical Research,vol.65,pp. 416-426,Apr. 2010.

[6] Wu Kang and Wei Yuchun, "The measure and analysis of regional development equilibrium of Jiangsu province since 1990s," Progress in Geography,vol.27,pp. 64-74,Jan. 2008.

[7] Han Jiafu,Zhang Zhong,Qi Qingwen, "Analysis and visualization of the uneven distribution of population in China," Geo-information Science,vol.9,pp. 14-19,Dec. 2007.

[8] Zhang Jingjing,Chen Zhengjiang, "Comprehensive research on space distribution of regional rural and urban population in the north slope of Tian Mountain," Journal of Arid Land Resources and Environment,vol.22,pp. 27-31,Jan. 2008.

[9] Statistics Bureau of Jiangsu Province, Jiangsu Statistical Yearbook,2004-2009. Beijing: China Statistics Press.

[10] Tong Xin, Population Sociology. Beijing: Peking University Press, 2001.

[11] Lu Anmin,Li Chengming,Lin Zongjian,and Wang jiayao, "An investigation on spatial continuous surface model of population density," Journal of Institute of Surveying and Mapping,vol.19,pp. 76-78,Mar. 2002.

[12] Sawada M.. Global Spatial Autocorrelation Indices - Moran's I, Geary's $\mathrm{C}$ and the General Cross-Product Statistic. http://www.lpc.uottawa.ca/publications/moransi/moran.htm.

[13] Yang Zhenshan and Cai Jianming, "Progress of spatial statistics and its application in economic geography," Progress in Geography,vol.29,pp. 757-768,June 2002.

[14] Zhang Shanyu. Introduction to the Population geography.Shanghai:East China Normal University Press,2007.

[15] Dobson,J.E.,Bright,E.A.,Coleman,P.R.,Durfee,R.C., and Worley,B.A., "A Global Population Database for Estimating Populations at Risk," Photogrammetric Engineering \& Remote Sensing, vol.66,pp. 849-857,2000.

[16] Qing Yaochen. Principle and application system model. Beijing:Science Press,2004.

[17] Deng Zutao,Lu Yuqi,“A study on urban population distribution and spatial auto-correlation in Yangtze Basin," Population and Economics, vol.66,pp. 7-12,2007. 